\title{
The Influence of Laser Beam on the Surface Integrity of Cutting Edge
}

Rastislav Nigrovič, Jozef Meško, Andrej Zrak

University of Zilina, Univerzitná 1, Department of Technological Engineering.E-mail: rastislav.nigrovic@fstroj.uniza.sk; jozef.mesko@fstroj.uniza.sk; andrej.zrak@fstroj.uniza.sk

The presented scientific article deals with cutting edges after cutting by laser beam. The article describes the characteristics of the laser beam the factors entering into the process of cutting by $\mathrm{CO}_{2}$ laser, and their interaction on the integrity of the cutting edge of selected technical materials - steel S23JR. The article includes experimental evaluation of the interaction of input factors and parameters on experimental samples with thickness $t=2 \mathrm{~mm}$ which were cut by applying different parameters for the accurate assessment of the impact for each selected technological parameters. The experimental part of this article deals with changes of speed laser cutting and cutting speed influence on HAZ width and hardness of cutting edges. The outcomes of this article may be the prediction and parameters settings recommended for laser cutting as regards of $\mathrm{HAZ}$ width and hardness of cutting edge.

Keywords: laser cutting, cutting parameters, cutting edge

\section{Acknowledgement}

This paper was elaborated under the project: KEGA no. 054 ŽU - 4/2012 and VEGA no.1/0186/09-Responsible investigator: prof. Ing. Jozef Meško, PhD.

\section{References}

[1] SILVFAST, W. T. (2004). Laser Fundamentals, Cambridge University Press, 666p. ISBN 0-521-83345-0.

[2] CARISTAN, L. C. (2004). Laser cutting guide for manufacturing. Dearborn, Michigan, USA: Society of Manufacturing Engineers, 452p. ISBN 0-87263-686-0.

[3] SVELTO, O. (2010). Principles of laser, Springer, 620p. ISBN 9781441913029.

[4] SEJC, P., BIELAK, R., SVEC, P., ROSKO, M. (2006). Computer simulation of heat affected zone during MIG brazing of zinc-coated steel sheets. In: Kovové materiály. Metallic materials. Vol. 44, No. 4, pp. 225-234. ISSN 0023-432X.

[5] KONAR, R., MICIAN, M., HLAVATY, I. (2014). Defect detection in pipelines during operation using Magnetic Flux Leakage and Phased Array ultrasonic method. In: Manufacturing technology, Vol. 14, No. 3, pp. $337-341$. J.E. Purkyne University, Ústi nad Labem. ISSN 1213-2489.

[6] KONAR, R., MICIAN, M. (2014). Non-destructive testing of welds in gas pipelines repairs with Phased Array ultrasonic technique. In: Manufacturing technology, Vol. 14, No. 1, pp. 42-47. J.E. Purkyne University, Ústi nad Labem. ISSN 1213-2489.

[7] MESKO, J., ZRAK, A., MULCZYK, K., TOFIL, S. (2014). Microstructure analysis of welded joints after laser welding. In: Manufacturing technology, Vol. 14. No. 3, pp. 355-359. 341. J.E. Purkyne University, Ústi nad Labem. ISSN 1213-2489.

[8] RADEK, N., MESKO, J., ZRAK, A. (2014). Technology of laser forming. In: Manufacturing technology, Vol. 14, No. 3, pp. 428-431. J.E. Purkyne University, Ústi nad Labem. ISSN 1213-2489.

[9] VRZGULA, P., FATURIK, M., MICIAN, M. (2014). New inspection technologies for identification of failure in the material and welded joints for area gas industry. In: Manufacturing technology, Vol. 14, No. 3, pp. 487-492. J.E. Purkyne University, Ústi nad Labem. ISSN 1213-2489.

[10] PATEK, M., KONAR, R., SLADEK, A., RADEK, N. (2014). Non-destructive testing of split sleeve welds by the ultrasonic TOFD method. In: Manufacturing technology, Vol. 14, No. 3, pp. 403-407. J.E. Purkyne University, Ústi nad Labem. ISSN 1213-2489.

[11] LAGO, J., BOKUVKA, O., NOVY, F. (2015). The weld toe improvement of Domex 700 by laser remelting. In: 32nd Danubia-Adria symposium on advances in experimental mechanics, pp. 142-143. University of Žilina, Žilina. ISBN 978-80-554-1094-4. 
[12] TRUMPF Werkzeugmaschinen GmbH + Co. KG. (2007). Technical information - Laser processing - CO2 laser. [online].2007,[cit.2015-04-27]. $<$ http://www.trumpf-laser.com/index.php?eID=tx_nawsecuredl\&u=0\&file=fileadmin/DAM/trumpflaser.com/Technische_Infos/TI_Laser_processing_CO2Lasers.PDF\& $\mathrm{t}=1431301513 \&$ hash $=\mathrm{b} 8 \mathrm{~b} 5 \mathrm{~d} 228412 \mathrm{cb} 1 \mathrm{cb} 218285732 \mathrm{c} 9 \mathrm{~d} 7 \mathrm{~b} 3 \mathrm{f}>$.

[13] TRULASER.(2008).[online].2014,[cit.2015-02-7]. $\quad<$ http://www.sk.trumpf.com/index.php?eID=tx_nawsecuredl\&u=0\&file=fileadmin/DAM/sk.trumpf.com/Prospekte/TruLaserSK.PDF\&t=1431272900\&hash $=\mathrm{e} 169 \mathrm{a} 4 \mathrm{de} 6 \mathrm{fcb} 624 \mathrm{~b} 457 \mathrm{f3} 8 \mathrm{c} 24 \mathrm{~b} 19 \mathrm{e} 246>$.

\section{Paper number: M2016246}

Copyright (C) 2016. Published by Manufacturing Technology. All rights reserved. 